\title{
Calculation of the Transverse Wake Function of a Highly Damped Periodic Structure
}

\author{
W. Wuensch, CERN, 1211 Geneva 23, Switzerland
}

\begin{abstract}
Higher order wakefields in the CLIC multibunch accelerating structure (called the TDS, Tapered Damped Structure) are suppressed through a combination of heavy damping and moderate detuning. A new approach to computing the transverse wake function of such a highly damped periodic structure is presented. The driving bunch produces fields that travel with the propagation characteristics (given by the frequency dependent complex wave number) of the damped periodic waveguide. The fields in the structure are calculated by integrating the propagated waves excited by the Fourier decomposed driving bunch. Strong damping produces a propagated wave integral that converges within a few cells. Computational and experimental techniques to obtain wave numbers are described.
\end{abstract}

\section{INTRODUCTION}

Higher order wakefields in the CLIC multibunch accelerating structure are suppressed through a combination of heavy damping by waveguides and moderate detuning. The techniques needed to compute the transverse wake function of a damped structure such as the TDS have been developed and include an uncoupled circuit model, a coupled double-band circuit model and MAFIA time domain computations [1,2,3]. The transverse wake functions of the TDS computed using these techniques agree very well and confirm that the structure achieves the required performance. None of the techniques, however, give a simple and intuitive understanding of the physics underlying the interaction between a relativistic beam and a heavily damped periodic structure. An attempt to clarify this has resulted in the formulation of a new method to determine the transverse wake.

This new method, named the "wave number" method, derives the wake directly from the propagation characteristics of fields inside a periodic structure and from the coupling of the fields to a small current slice. The derivation is made in the frequency domain, giving the transverse kick spectrum, and the time domain wake function is found by taking the Fourier transform. The propagation characteristics and coupling can be obtained simply from either measurement or computation.

The wave number method naturally takes into account such effects as the cutoff frequency of damping waveguides and imperfect waveguide terminations. The concept of synchronism, confusing in the presence of heavy damping, emerges naturally from the derivation. The wake can be calculated over an arbitrary frequency band so higher passbands can be included without any reformulation as is necessary in a circuit model.

\section{DERIVATION OF THE KICK SPECTRUM}

The derivation of the transverse kick spectrum is made in the following steps: First the bunch driving the wake is Fourier decomposed into a steady state current distribution. Next the waves radiated by this current distribution are described and propagation is included. An integral is then made which sums the fields that propagate to a point in the structure from all other points in the structure. These fields give the kick spectrum.

A very short driving bunch that travels to the right (towards $+z$ ) at the speed of light and which crosses $z=0$ at $t=0$ is described by the delta function current,

$$
I(z, t)=\delta(z-c t)
$$

The Fourier transform of this driving current is simply,

$$
\begin{aligned}
I(z, \omega) & =\int_{-\infty}^{\infty} \delta(z-c t) e^{i \omega t} d t \\
& =e^{i z \frac{\omega}{c}}
\end{aligned}
$$

This represents a flat spectrum of currents, each frequency with a spatial variation given by the wave number $k_{\text {beam }}=\omega / c$.

Every differential slice of current acts as a small antenna launching fields that travel to the right and left inside the waveguide. The coupling of the current slice to the fields is a function of frequency, since the field pattern of a mode changes with frequency, and is represented by the term $A(\omega)$. The phase of the radiated wave is determined by the phase of the current slice. The phasors of the waves radiated by the current distribution at the position they are radiated are consequently,

$$
A(\omega) e^{i z \frac{\omega}{c}}
$$

Depending on the direction, the waves then propagate according to the wave number,

$$
\begin{array}{ll}
e^{i k(\omega) z} & \text { to the right } \\
e^{-i k(\omega) z} & \text { to the left }
\end{array}
$$


A schematic picture of the fields that fall on the origin which have been radiated from reference distances $-\mathrm{z}_{0}$ and $\mathrm{z}_{0}$ is shown in Figure 1.

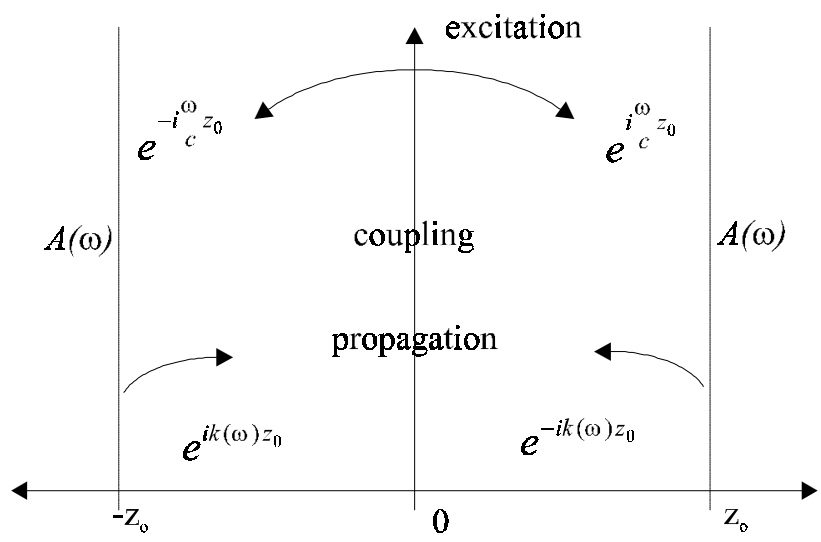

Figure 1: Schematic picture of the excitation and propagation of waves inside the waveguide.

The phasors of the wave radiated from the positions $\pm z_{0}$ and arriving at the origin are found by multiplying the phasor of the excitation by that of the propagation. The waves travelling to the right from $-z_{0}$ are given by,

$$
e^{-i \frac{\omega}{c} z_{0}} A(\omega) e^{i k(\omega)\left(z+z_{0}\right)}=e^{-i \frac{\omega}{c} z_{0}} A(\omega) e^{i k(\omega) z_{0}}(5)
$$

The waves travelling to the left from $z_{0}$ are,

$$
e^{i \frac{\omega}{c} z_{0}} A(\omega) e^{-i k(\omega)\left(z-z_{0}\right)}=e^{i \frac{\omega}{c} z_{0}} A(\omega) e^{i k(\omega) z_{0}}
$$

The total field at the origin is the integral of the phasors radiated from all distances,

$$
\begin{aligned}
E(\omega) & =A(\omega) \int_{0}^{\infty}\left(e^{i\left(k(\omega)-\frac{\omega}{c}\right) z_{0}}+e^{i\left(k(\omega)+\frac{\omega}{c}\right) z_{0}}\right) d z_{0} \\
& =A(\omega)\left[\frac{e^{i\left(k(\omega)-\frac{\omega}{c}\right) z_{0}}}{i\left(k(\omega)-\frac{\omega}{c}\right)}+\frac{e^{i\left(k(\omega)+\frac{\omega}{c}\right) z_{0}}}{i\left(k(\omega)+\frac{\omega}{c}\right)}\right]_{0}^{\infty}
\end{aligned}
$$

If there are any losses, $k(\omega)$ is complex and the integral converges,

$$
\begin{aligned}
E(\omega)= & i A(\omega)\left(\frac{1}{k(\omega)-\frac{\omega}{c}}+\frac{1}{k(\omega)+\frac{\omega}{c}}\right) \\
= & \frac{2 i k(\omega) A(\omega)}{k^{2}(\omega)-\left(\frac{\omega}{c}\right)^{2}}
\end{aligned}
$$

The kick on the beam is derived from the field by multiplying by the same factor $A(\omega)$ as the coupling of the current to the field and is given by,

$$
\operatorname{Kick}(\omega)=\frac{2 i k(\omega) A^{2}(\omega)}{k^{2}(\omega)-\left(\frac{\omega}{c}\right)^{2}}
$$

Equation 8. shows that the kick spectrum, and consequently the transverse wake function, is a remarkably simple function of only a wave number spectrum and a normalized frequency dependant coupling. Equation 8. is referred to as the 'propagated wave integral'.

\section{INTERPRETATION OF THE DERIVATION}

A number of physical effects emerge from the derivation. Synchronism between the relativistic beam and the structure's phase velocity is evident in equation (8). A low loss mode has a wave number that is predominantly real and there is a strong peak in the spectrum $E(\omega)$ for the frequency where the real part of $k(\omega)$ is equal to $\omega / c$. The peak occurs in the integral of the fields generated behind the exciting bunch (left hand term in equation 7), because the radiated waves from behind the bunch add constructively over large distances. A narrow peak dominates the kick spectrum and results in a strong wake at the synchronous frequency.

In a damped structure, the imaginary part of $k(\omega)$ is larger compared to the real part so the denominator in equation (8) does not become small when the real part equals $\omega / \mathrm{c}$. The kick spectrum is broadened, resulting in the expected decay when the Fourier transform is taken.

From another perspective, fields in a damped structure are attenuated with distance causing a suppression of the constructive addition of fields over long distances that otherwise leads to a sharply peaked wake spectrum. The interaction between beam and structure occurs only over distances of the order of the attenuation length. This emerges in equation (7), where the integral converges more quickly for increasing losses. A convenient side effect is that short models can be used for computations and measurements on heavily damped structures.

The derivation given is section 2 has implicitly assumed that the interaction with the mode is dominated by interaction with the lowest space harmonic. A higher space harmonic can be considered separately, and the kick spectrum added to that of the lowest space harmonic. All space harmonics of course have the same value of complex wave number. 


\section{PRINCIPLES OF MEASUREMENT AND COMPUTATION}

The derivation of the kick spectrum has shown that the wakefield behavior of a structure can be computed from a simple function of only $k(\omega)$ and $A(\omega)$. These two terms are readily determined for a particular structure geometry using both measurement and computer modeling. Both measurement and computer modeling are simplified by including a conducting azimuthal symmetry plane: dipole modes are supported and monopolar modes are not. Measurement will be considered first.

The most conceptually simple measurement of $k(\omega)$ in a periodic structure can be made by measuring the transmission through two lengths of guide that differ by one structure period (with period length $l$ ). The change in transmission directly gives $\mathrm{e}^{\mathrm{i} k(\omega) l}$. Good accuracy requires a good match between the periodic structure and the measuring waveguide. The damping inherent to a high loss structure like the TDS can be used to eliminate multiple reflections and the measurement can be made on a relatively short section of line with large reflections into and out of the periodic line.

$A(\omega)$ can be measured in an experimental setup using small loop antennas because an offset beam excites dipole modes with the same sensitivity to field configuration, and consequently frequency variation, as a small loop antenna. An offset beam excites dipole modes equivalently to two beams traveling in opposite directions and offset in opposite directions. A pair of offset beams can be decomposed into a series of infinitesimal current loops. Currents flowing between the beams cancel in adjacent loops. A schematic representation of this is shown in Figure 2. These current loops have the same topology and thus the same field pattern sensitivity as a loop antenna.

\section{exciting current}

\section{symmetry axis}

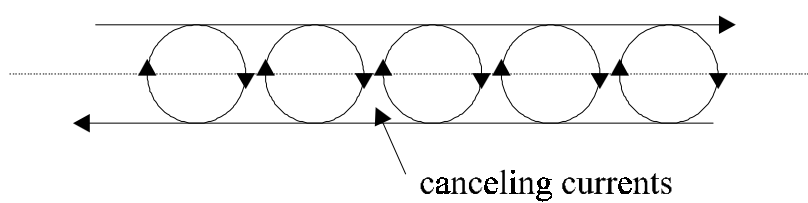

Figure 2: Equivalence between the driving beam and a decomposition in current loops.

$A(\omega)$ and $k(\omega)$ can be simultaneously extracted from a short model shown schematically in Figure 3. The model has a conducting azimuthal symmetry plane and a pair of small half loops mounted on the end of a coaxial cable. The measurement consists of comparing the transmission across one cell, $T_{1}$, to the transmission across two cells, $T_{2}$.

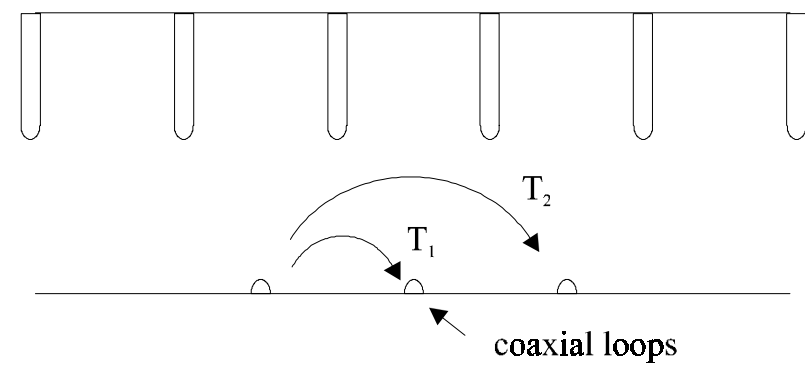

Figure 3: Schematic view for the measurement of $A(\omega)$ and $k(\omega)$.

The two transmission coefficient can be expressed in terms of $A(\omega)$ and $k(\omega)$,

$$
\begin{aligned}
& T_{1}=A^{2}(\omega) e^{i k(\omega) l} \\
& T_{2}=A^{2}(\omega) e^{i 2 k(\omega) l}
\end{aligned}
$$

Thus,

$$
\begin{aligned}
& A^{2}(\omega)=\frac{T_{1}^{2}}{T_{2}} \\
& k(\omega)=\frac{1}{i l} \ln \left(\frac{T_{2}}{T_{1}}\right)
\end{aligned}
$$

This measurement is in principle possible with any periodic structure although the ends of a low-loss structure must be well matched to avoid creating standing waves. The ends of a highly-damped structure are effectively matched by the internal attenuation over a sufficient length. The loops must be small compared to about a quarter of the free space wavelength at the frequency of measurement.

The measurement techniques presented above are also easily transferable to computer programs, such as HFSS, that are able to compute scattering matrices. In this way the intermediate results in the computation of a transverse wake function can be compared directly to the results from simple experiments.

\section{REFERENCES}

[1] M. Dehler, "Modelling a $30 \mathrm{GHz}$ Waveguide Loaded Detuned Structure for the Compact Linear Collider (CLIC)", CERN PS/RF/Note 98-09.

[2] M. Dehler, I. Wilson, W. Wuensch, "A Tapered Damped Accelerating Structure for CLIC", Proc. LINAC 98, Chicago, August 1998.

[3] M. Dehler, I. Wilson, W. Wuensch, "CLIC Waveuide Damped Accelerating Structure Studies", Proc. Linac 96, Geneva, August 1998. 